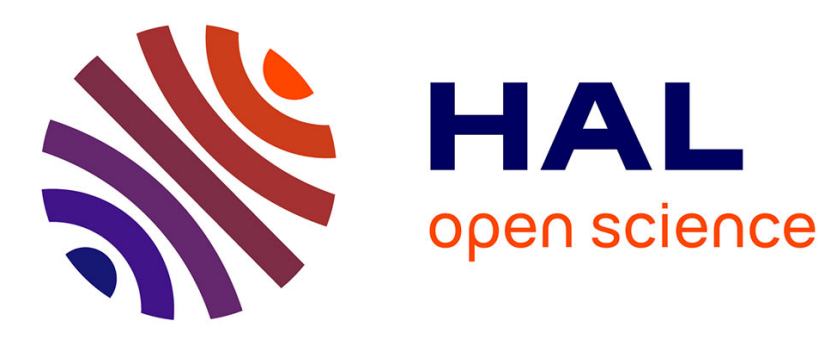

\title{
IMS-based distributed multimedia conferencing service for LTE
}

\author{
Tien A. Le, Hang Nguyen, Noel Crespi
}

\section{To cite this version:}

Tien A. Le, Hang Nguyen, Noel Crespi. IMS-based distributed multimedia conferencing service for LTE. 2012 IEEE Wireless Communications and Networking Conference: Services, Applications, and Business (IEEE WCNC 2012 Track 4 SAB), citeulike-article-id = 10124423, Apr 2012, Paris, France, France. pp.1-6. hal-00703023

\section{HAL Id: hal-00703023 https://hal.science/hal-00703023}

Submitted on 31 May 2012

HAL is a multi-disciplinary open access archive for the deposit and dissemination of scientific research documents, whether they are published or not. The documents may come from teaching and research institutions in France or abroad, or from public or private research centers.
L'archive ouverte pluridisciplinaire HAL, est destinée au dépôt et à la diffusion de documents scientifiques de niveau recherche, publiés ou non, émanant des établissements d'enseignement et de recherche français ou étrangers, des laboratoires publics ou privés. 


\title{
IMS-based distributed multimedia conferencing service for LTE
}

\author{
Tien Anh Le, Hang Nguyen, Noel Crespi
}

\begin{abstract}
This research proposes a new architecture for the inter-connectivity between UEs running on the LTE infrastructure participating in an Application Layer Multicast-based distributed conference. The main contribution is that the proposal replaces the standard centralized architecture of the IMS-based conference with a more robust solution utilizing intelligence and computational capacity of LTE's eNodeBs. The costly Media Resource Function Controller (MRFC) can be fully omitted from the IMS without effecting the conference. A prototype has been built to prove the feasibility of the proposed architecture and evaluate its performances.
\end{abstract}

Index Terms - distributed video conference; service architecture; IMS; LTE; ALM

\section{INTRODUCTION}

Video conferencing service is the most complex type of video communications. There are two main types of video conferencing service architectures: centralized architecture using Multipoint Control Unit (MCU) and distributed architecture using multicast mechanism. The centralized architecture has many disadvantages such as cost (incapable of neither increasing the Capital Expenditures or CAPEX nor lowering Operational Expenditure or OPEX ) or a very high delay especially when the number of participants increases. The distributed architecture is however not yet mature. Its advantage is that it aims at distributing the computational loads of the conference session to all participants based on their capabilities.

Recently, mobile participants have been equipped with high computational devices using radio access networks (e.g. 4G: Long Term Evolution, WiMAX) with extra bandwidth to contribute in a distributed conference. In this research work, the LTE network is used as a demonstration of a $4 \mathrm{G}$ infrastructure.

The main problem tackled in this paper is: the distributed video conferencing service architecture and the $4 \mathrm{G}$ network architecture have been designed separately without considering the other's requirements. The $4 \mathrm{G}$ network relies on the IP Multimedia Subsystem (IMS[1]) to provide multimedia services including video conference. Nevertheless, the IMS-based video conferencing service is built and standardized for MCU-based or centralized service architecture. Therefore, it shares many similar problems with the centralized video conferencing service architecture[2], especially when the number of User Equipments (UE) who participate in the conference via the $4 \mathrm{G}$ infrastructure increases.

In this research work, a new solution is proposed so that the current IMS-based LTE infrastructure can seamlessly support distributed video conferencing services. The main contributions of the research are:

- Propose an IMS-based architecture for support-

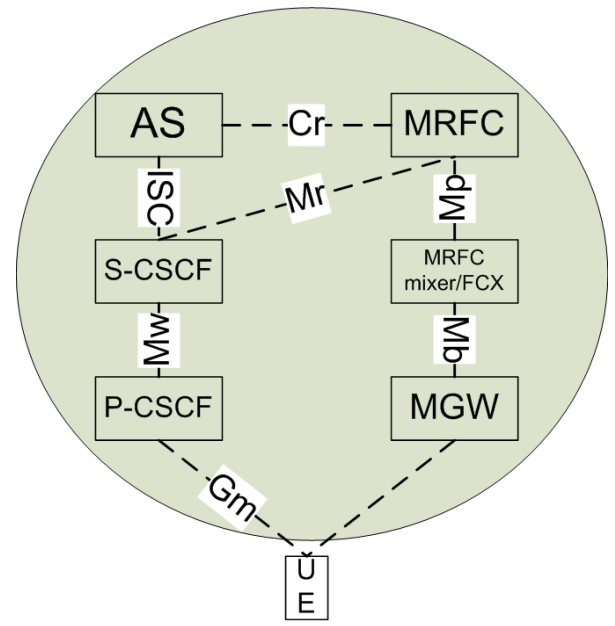

Fig. 1. 3GPP IMS conference architecture[3]

ing LTE's UEs to participate in distributed scalable video conferencing service without using a centralized MCU,

- Develop a proof-of-concept prototype to prove the feasibility and compatibility of the newly proposed solution,

- Evaluate the performance of the proposed system under realistic working scenarios.

\section{3GPP IMS CONFERENCE ARCHITECTURE}

\section{A. 3GPP IMS conference architecture}

Fig.1 shows the 3GPP standard architecture for the IMS-based conference architecture. Here Session Initiation Protocol (SIP) and Real-time Transfer Protocol (RTP) are used as the main signaling and media transportation protocols. The call session control functions (CSCFs) are entities that route SIP messages. The media gateway (MGW) is the entity that handles/forwards RTP traffic down to the UE when necessary. The conference focus is in the Media Resource Function Controller (MRFC) and in the conferencing AS. The MRFP provides all of the media related functions (e.g., mixing, transcoding, transrating...) required for conferencing. It may also contain a Floor Control Server (FCS) function. Since all the media related functions for conferencing are done at the MRFP, this architecture is highly centralized. To overcome the disadvantages of the centralized architecture, several proposals have been made in order to support distributed conferencing service architecture on the current centralized architecture of IMS-base conferencing service on LTE network. In[4], the FCS feature is proposed to be separated from the MRFP. However, that does not change the centralized nature of the 
architecture. $\operatorname{In}[5]$, a distributed solution is proposed as an overlay network of centralized conferencing clouds. However, the proposed architecture does not provide a proper integration with any specific overlay algorithm. Moreover, even-though a Content Distributed Network (CDN) with proxy servers has been constructed to support the integration, the clients still have to process all of the signaling and media loads. The proxy servers serve mainly as proxy MCUs to connect several clients together using a centralized architecture and then connect all of those centralized groups together by creating an overlay of proxy servers. To conclude, the conventional methods for IMS-based video conferencing services are either centralized or have not fully utilized the capacity of the $4 \mathrm{G}$ infrastructure.

\section{IMS-BASED DISTRIBUTED VIDEO CONFERENCING SERVICE}

As a many-to-many communication mechanism, distributed video conferencing services are mainly built on multicast. IP-multicast [6] is so far the most efficient type of multicast. However, deploying problems are preventing it from being widely applied[7]. Application Layer Multicast (ALM) is a promising alternative. Many ALM algorithms have been proposed and distributed video conferencing services have been built upon ALM[8].

The problem is, since ALM algorithms are working on the application layer, it does not care which kind of access network has been used by the terminals in order to participate in the conference session. Nevertheless, many participants are using a radio access network (such as LTE) to join the conference. Thus, sometimes the limited resources of the mobile terminals and the radio channels are unnecessarily used up by ALM's operations such as heart-beating, data forwarding.

LTE applies a ring-topology[9] where components connect together using the same core network. All eNodeBs in LTE are smart Base Station Systems (BSS) which are capable of contributing computational capacity to the service[10]. If these eNodeBs can represent UEs in handling ALM's data traffic forwarding and control message processing, the UEs can participate in the distributed conference as if they are participating in the conventional IMS-based centralized conference.

While the distributed conferencing service architecture can overcome many technical limitations of the centralized architecture, the business model of the distributed conferencing architecture can create a win-win services for participants in which they can contribute their computation and get the service free or they can even contribute their computation for money. Meanwhile, the distribute architecture can still support the existing business model which is providing by the centralized architecture.

\section{A. Design requirements}

The main target of our research is to provide an interconnectivity between the distributed video conferencing service based on ALM overlay network and mobile terminals participating in the conferencing by using the LTE in-

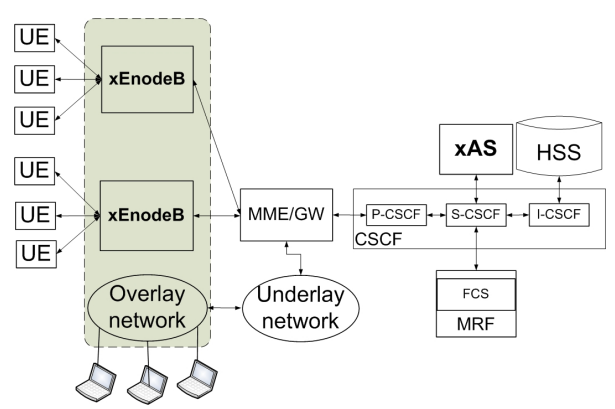

Fig. 2. LTE IMS-based distributed conference.

frastructure. The inter-connectivity solution is built based on IMS. The main requirements are:

- Utilize available resource and information which can be easily obtained from the LTE infrastructure to contribute in the ALM-based distributed conference so that the limited resources of the mobile terminals are efficiently used,

- Discard the standard centralized architecture using MRFC in order to reduce the total expense of the entire solution, avoid the single point of failure while still maintain conferencing features,

- Apply floor control mechanism from the ALM-based conferencing architecture,

- Support a seamless integration among the LTE mobile terminals and the ALM-based conferencing platform during a mobile video conference session such as join/leave, pause/return, soft hand-over, heartbeat,

- Provide a QoS-guaranteed mechanism for QoSrequired ALM conferencing architecture.

\section{B. Proposal of IMS-based LTE distributed conferencing service}

In this proposal, eNodeBs are used as the proxy servers for bridging between the participating UEs and the distributed conference. An AS (Application Server) will mainly be used to manage the floor control and the mapping between UEs and their proxy eNodeBs. We call the extended version of eNodeB, supporting the ALM's protocol, XeNodeB, and the extended version of the AS - xAS. The feasibility of using eNodeB for advanced features has been investigated in[11][12]. Follows are the main features of the XeNodedB and xAS.

Extended features of the XeNodeB:

- Participate as a node in the Overlays network,

- Transfer or forward data and control messages,

- Scalable Video layer registration.

To achieve these features, each XeNodeB manages:

- Routing tables:

- Participating list of UEs which are being served by that XeNodeB. The list contains the UE's IP, Overlay role (source/relay/forwarding), status (idle/active), registered video layer(s) (base/enhanced),

- Report to the xAS about any change in status (idle/out/active) of its managing UEs. 
- List of peers on the distributed conference containing the peer's IP, distance (cost to reach that peer from the current XeNodeB).

- Event Processor:

- Update the participant list in join/leave, pause/return, hand-over operations,

- Report to xAS about the status (idle/left/active) of its managing UEs.

- Overlay interface:

- Interface between its participating UEs and the overlay network,

- Filter forwarding packets and send them back to the overlay network to save UEs capacity.

- Signal processor:

- Obtain UEs status (availability, connection QoS) from xAS,

- Send back UEs status to the distributed conference via the overlay interface when required.

- QoS Video Layer registration: Check the UEs available QoS and its registered video layers if they can match together.

Extended features of the $\mathbf{x A S}$ :

- Floor control for the video conferencing application: conference ID, participant's list, participant's status,

- Obtain UE-related information from the LTE network and forward to the overlaying nodes,

- QoS guarantee, conference's QoS policies.

To achieve these features, each xAS manages:

- Conference list: Contain conference ID, IP, XeNode ID, Conference status (in progress/terminated),

- Signaling processor:

- Receive requests from XeNodeB's Signaling Processor,

- Interrogate UEs information (QoS, availability...) from the Home Subscriber Server (HSS) and IMS and send back to XeNodeB's Signaling Processor,

- Event processor:

- Receive updates from XeNodeB's Event Processor about leave/join, pause/return, hand-over,

- Update the Conference list,

- Conference policy:

- Starting time, duration, maximum number of participants,

- QoS requirements,

- Billing information.

Fig. 3 shows the call flow when an UE wants to participate in or leave a distributed video conference. First, when turned on, it automatically sends the REGISTER message to its XeNodeB. The XeNodeB then updates its routing table and sends (eNodeB - ID,UE - SIP - Account) to the xAS. When received, this information will be stored in the xAS's Conference List. When the UE wants to initiates its participation in a distributed conference, it sends the INVITE(Conference ID, Layer Registration) message, containing the maximum number of enhancement layer(s) it wants to receive from the conference multicast tree, to the controlling XeNodeB. The XeNodeB has to convert the number of layer(s) to a QoS parameter which

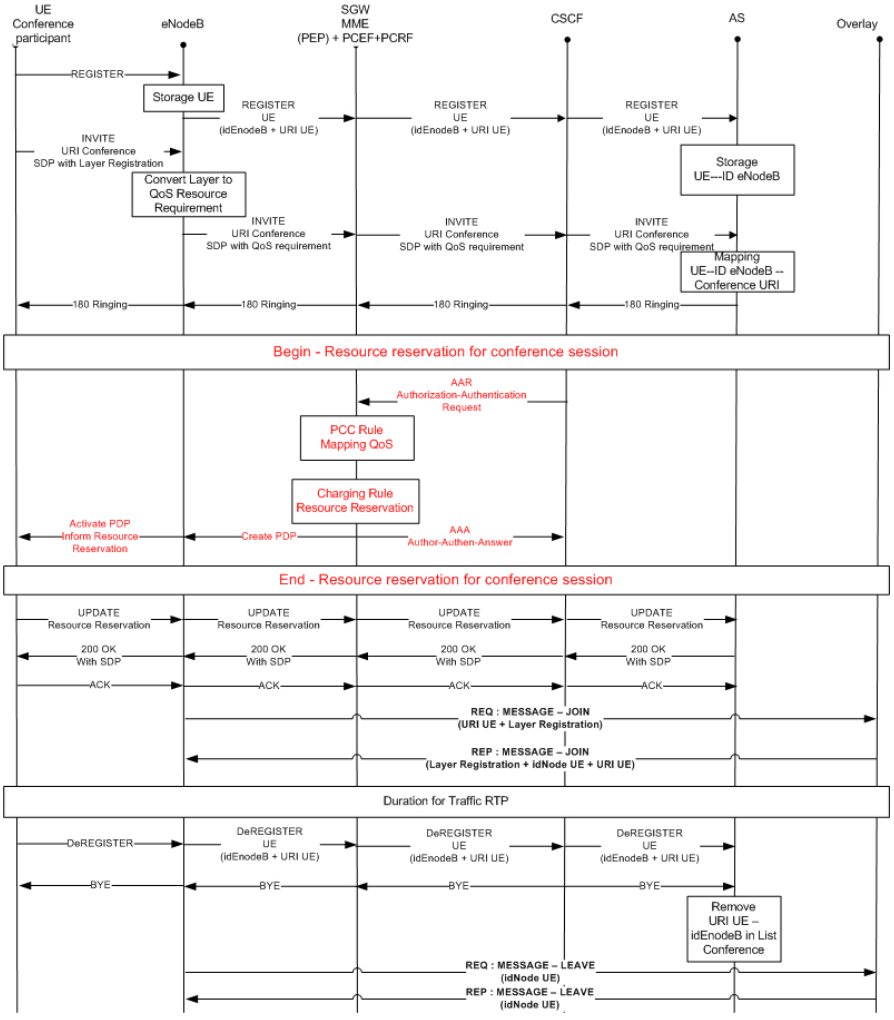

Fig. 3. Join/Leave of the UE to/from distributed conference with QoS support.

is understandable by the Policy Charging Rule Function (PCRF). Next, the INVITE message is forwarded to the xAS for mapping among the Conference-ID, UE-SIPAccount, and XeNodeB-ID in the xAS's conference list. At the same time, the INVITE message is forwarded to the Policy Charging Enforcement Function (PCEF) using the Authorization-Authentication Request (AAR) message by the Call Session Control Function (CSCF). The PCRF will check whether the UE has subscribed for enough QoS resource in order to receive the required number of enhancement layers. If the subscription is enough, a resource reservation request will be sent to the $\mathrm{PCEF}$ to activate the resource policy for the UE to join the distributed conference[13]. The confirm resource is then reported to the xAS for updating the QoS requirements, and billing information of the Conference Policy[12]. After receiving the QoS confirmation, the XeNodeB sends an ALM's JOINREQ(UE-SIP-Account, Layer-Reg) message to the ALM group to represent the UE participating in the ALM tree. After a new node has been successfully added to the ALM tree, a JOIN-REP(UE-ID, UE-SIP-Account, Lay-Reg) is responded to the XeNodeB. The UE-ID is assigned by the ALM and reported to the XeNodeB for managing purposes. When the UE wants to leave the conference, a DeEGISTER(eNodeB-ID, UE-SIP-Account) is sent to the xAS and then a REQ-LEAVE(UE-ID) is sent to the ALM tree for leaving request. The UE's record is then removed from the xAS's Conference List. Fig.4 illustrates the Pause/Return operations of an UE over the distributed 


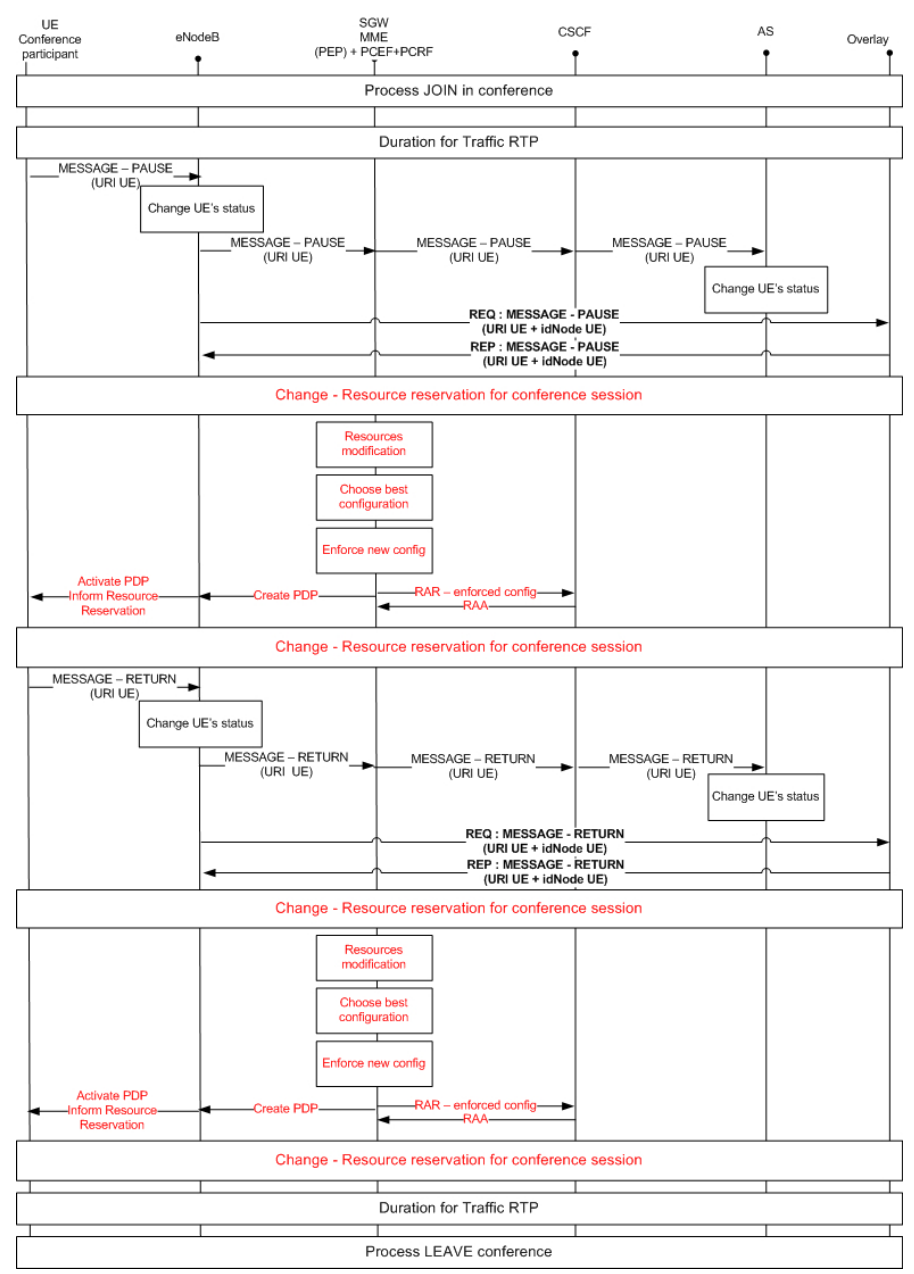

Fig. 4. Pause/Return operations over the distributed video conferencing service with QoS support.

video conference service. During the conference session, the UE may want to suspend the service (it does not want to receive, transmit video streams and signaling messages for a while) but still wants to return to the ALM group with the smallest delay afterward. Of course, if the suspending time is too long, the UE will be discarded from the ALM group. When the UE wants to become idle, it sends the PAUSE(UE-SIP-Account) to the XeNodeB and the xAS for them to update their lists. Then, the XeNodeB automatically sends a PAUSE-REQ(UE-ID) to the ALM group. The ALM group will stops sending bit-streams and signaling messages(HEARTBEAT) to the specific UE-ID so that the UE will be placed in the waiting list of the ALM group within a certain time. If the UE returns to the conference within that time, it will only have to send a RETURN(UESIP-Account, UE-ID) to the RDV point of the ALM group and then come back to the ALM tree. If the UE is idle for too long, it will be automatically discarded from the waiting list of the ALM group. When working in the idle mode, the UE give back its reserved resource to the network via a Resource Modification process. The resource will be given back to the UE when it returns. Fig.5 shows an UE going through a hand-over process while participating in the dis-

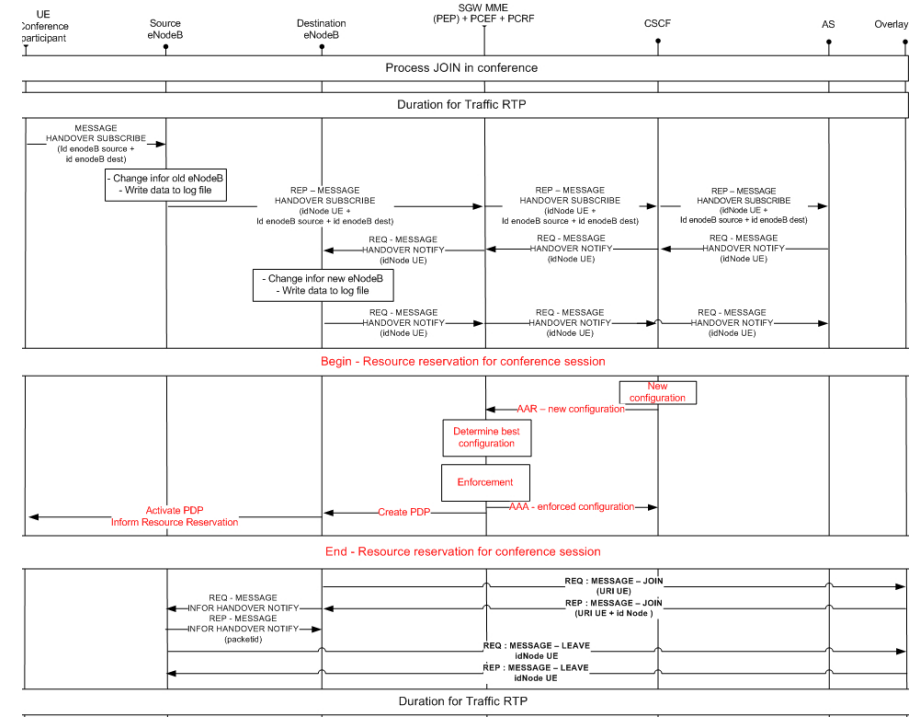

Fig. 5. Handover operation when participating in a distributed conference with QoS support.

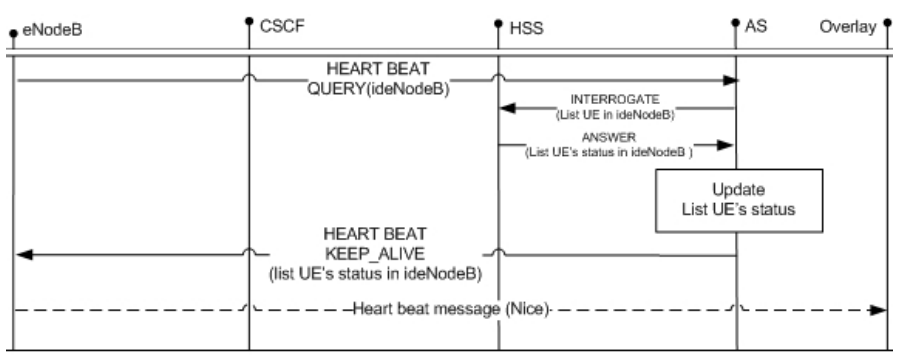

Fig. 6. Handling heartbeat operation in a distributed conference.

tributed conference. When an UE is about to be handed over from the source to the destination XeNodeB, it sends HANDOVER-SUBSCRIBE(Src-eNodeB-ID, Des-eNodeBID) to the xAS via the source XeNodeB. The xAS will then send the HANDOVER-NOTIFY(UE-ID) to the destination XeNodeB to notify it about a service hand-over request. A new Resource Reservation process is made for the UE to be attached to the new eNodeB. The Destination XeNodeB then sends a JOIN message to the ALM group to create another UE-ID for the same UE. At this time, two representing nodes are maintained by the two XeNodeBs in the ALM group so that a soft hand-over process is obtained. The two of-the-same nodes are sending and receiving bit-streams as any other normal node in the ALM group. It will help the UE to maintain its conference service via both eNodeBs before the old connection is actually broken. The source XeNodeB sends a LEAVE-REQ message to the ALM group and waits to receive the LEAVEREP from the ALM group. Afterward, the old UE-ID is removed from the ALM group and only one UE-ID will represent the UE in the conference. Fig. 6 shows the heartbeat handling process in the distributed conference with the support of the HSS. According to the fact that, many ALM algorithms have to depend on a heartbeat mechanism to maintain their group. If the UE has to directly respond to all heartbeats, it will soon run out of power and com- 
putational capacity. Meanwhile, all information regarding an UE availability is available at the HSS, a simple HSS interrogation operation trigger by the AS can solve this problem. Therefore, the XeNodeB will periodically send a HEARTBEAT-QUERY message to the xAS asking for the availability of all UEs which are joining the distributed conference under its representative. When this message is received at the xAS, all UE-IDs are obtained from the Conference List. Next, an HSS-INTERROGATION-REQ(UEIDs) message containing a list of all UE controlling by the originating XeNodeB will be sent to the HSS. The HSS will send back the availability information of all requesting UE obtained from the network to the xAS for it to forward to the XeNodeB. The XeNodeB will then send back the heartbeats of all the UE it is representing to the ALM group to inform it about their living. For floor control purposes, the ALM-based distributed conference will update the list of all peers participating in the conference from the ALM group to the xAS.

\section{PRototype And Evaluation Results}

In order to evaluate the proposal's performance and its feasibility, a prototype has been developed. The prototype is publicly available at[14]. Fig.7 shows the architecture of the prototype in which 4 UEs are participating into an ALM-based video conferencing service using a LTE infrastructure with IMS support. We used OpenIMS[15] as the IMS core and the Mobicents platform to build the xAS and XeNodeB. A distributed conferencing service is built based on[2]. A RDV point in the overlay and all XeNodeBs are equipped with a SIP interface so that the overlay can communicate with the xAS via the IMS core. We have built 3 evaluation scenarios running with different numbers of participants in which 4 UEs are participating via the IMS core:

- Scenario 1: Centralized IMS-based video conference using MRFP as recommended,

- Scenario 2: IMS-based distributed video conferencing service for LTE networks,

- Scenario 3: Pure ALM-based distributed video conferencing service.

Scenario 3 can be referred to as the Web-NGN converged multimedia conferencing system. The most troublesome problem of this scenario (as to be further investigated in the evaluation) is that, the mobile terminals have to handle all unnecessary singling and data forwarding traffic as any other peer in the ALM group (many of them may be work stations with at least unlimited power). Therefore, their limited power will be dried up rapidly and their poor radio resource will be filled up mostly by the ALM's unnecessary singling and data forwarding traffic. In all scenarios, for audio conference, each peer sends 100 audio packets, for video conference, each peer sends 300 video frames. Data has been measured 3 times for each number of participants in each scenario for convergence. Fig. 8 and Fig.9 show the average number of signaling messages calculated at UE's interface during an audio/video conferencing session of scenarios 2 and 3 . The result shows that scenario 3 has to use

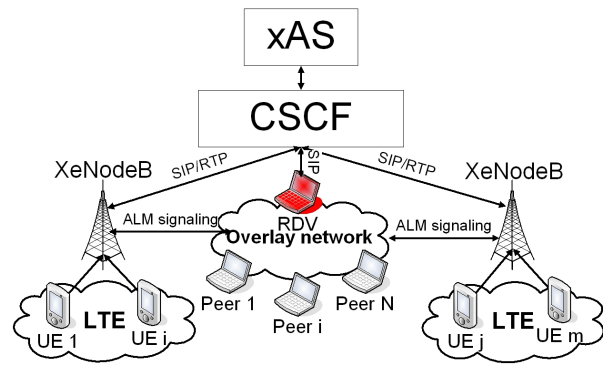

Fig. 7. Architecture of the prototype.

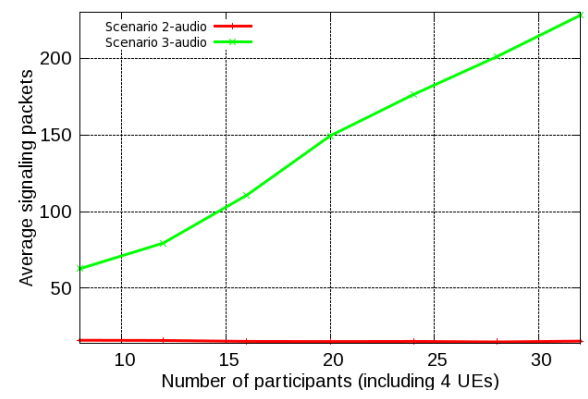

Fig. 8. Average number of signaling messages required during an audio conferencing session of scenarios 2 and 3 .

much more signaling packets than scenario 2 for maintaining the distributed conference. It is because most of the signaling loads have been processed by the XeNodeBs.

Fig.10 and Fig.11 show the comparison between the average data traffic monitored on UEs in scenarios 2 and 3 for an audio/video conference. Apparently, data traffic in scenario 2 is lower than in scenario 3 since the $\mathrm{XeN}$ odeBs have automatically routed forwarding traffic for its managing UEs in the overlay. Therefore, UEs only have to process the data traffic which is intentionally sent to them. Fig.12 shows that scenario 1 and 2 require a similar number of signaling packets on UEs. Fig.13 shows that, the average data traffic at an UE in scenario 2 is slightly higher than UE's average traffic in scenario 1 but far less than the MCU's average traffic in scenario 1.

\section{Conclusion}

This research proposes a new architecture for the interconnectivity between UEs running on the LTE infrastruc-

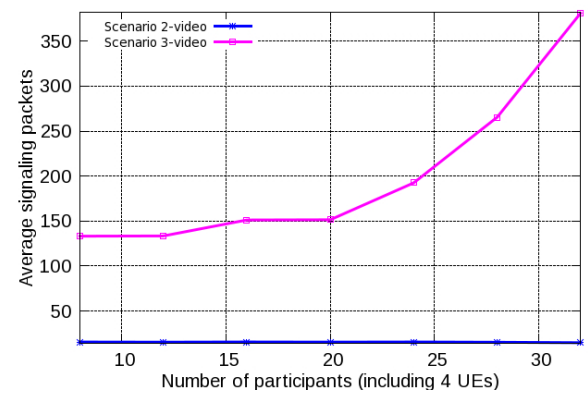

Fig. 9. Average number of signaling messages required during an video conferencing session of scenarios 2 and 3. 


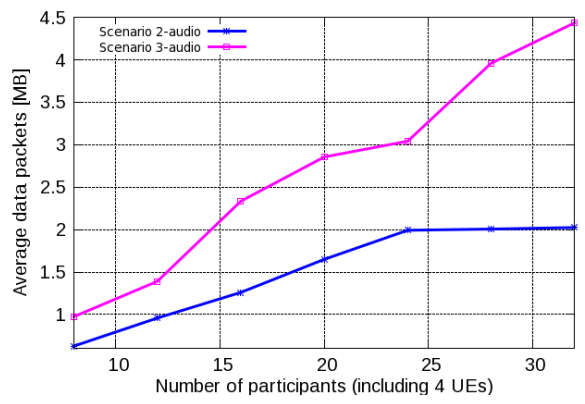

Fig. 10. Average number of data packets sent/received at each participant during an audio conferencing session of scenarios 2 and 3 .

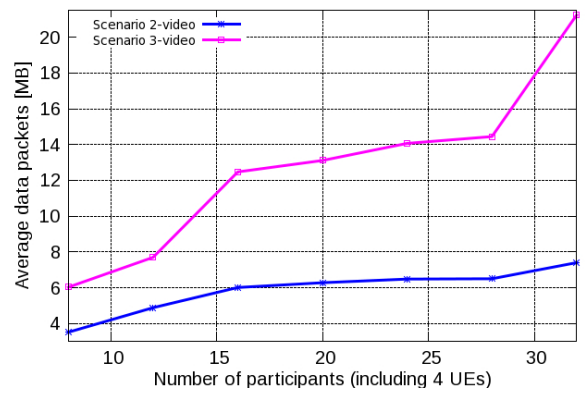

Fig. 11. Average number of data packets sent/received at each participant during an video conferencing session of scenarios 2 and 3.

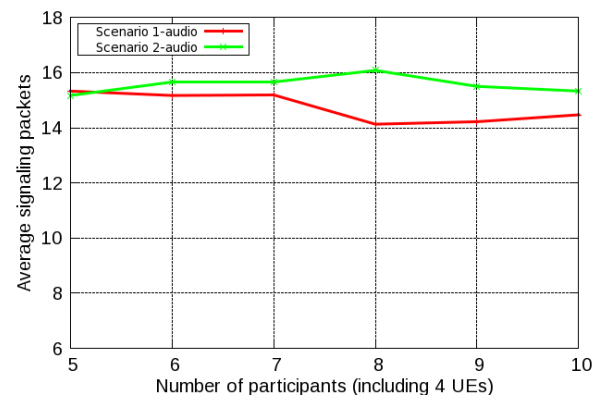

Fig. 12. Average number of signaling messages required during an audio conferencing session of scenarios 1 and 2 .

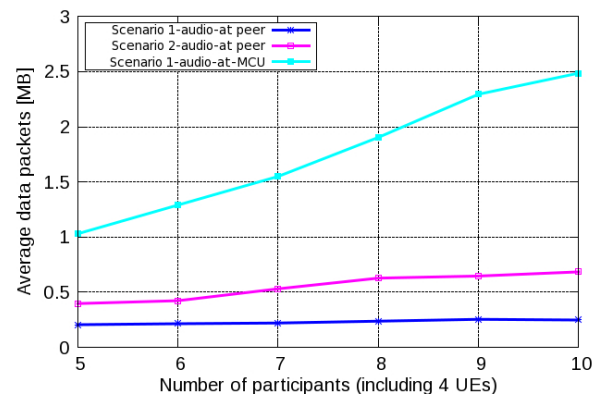

Fig. 13. Average number of data traffic at each participant/MCU during an audio conferencing session of scenarios 1 and 2 . ture participating in an overlay-based distributed conference. Experimental results carried on prototype have shown a great reduce in signalling traffic as well as data traffic handled by each UE and the core network. Various conferencing scenarios such as join/leave, pause/return, handover, heartbeat have been considered in the prototype. The main contribution is that the proposal replaces the standard centralized architecture of the IMSbased conference by a more robust solution utilizing intelligence and computational capacity of LTE's eNodeBs. A distributed architecture for a multimedia service has been supported well by the LTE network in the proposal. Its distributed nature bring a great reduce in cost and more flexibility to the multimedia conference service. The architecture can also be applied in WiMAX network with some possible modifications. The security of the proposal can be inherited from the authorization and encryption mechanisms applied by LTE and SIP technologies.

\section{Acknowledgement}

The authors are grateful to Quoc Tuan Nguyen, Quang Hoang Nguyen and Hongguang Zhang for their important contributions in building the prototype for the proposal.

\section{REFERENCES}

[1] 3rd Generation Partnership Project, "IP Multimedia Subsystem (IMS), Stage 2 (Release 5)," 3GPP TS 23.228, vol. 5, 2004.

[2] Tien A. Le and Hang Nguyen, "Centralized and distributed architectures of scalable video conferencing services," in The Second International Conference on Ubiquitous and Future Networks (ICUFN 2010), Jeju Island, Korea, June 2010, pp. 394399.

[3] 3gpp, "Conferencing using the ip multimedia (im) core network (cn) subsystem," Tech. Rep., March 2008.

[4] F. Belqasmi, C. Fu, M. Alrubaye, and R. Glitho, "Design and implementation of advanced multimedia conferencing applications in the 3GPP IP multimedia subsystem," IEEE Communications Magazine, vol. 47, no. 11, pp. 156-163, 2009.

[5] A. Buono, S. Loreto, L. Miniero, and S. P. Romano, "A distributed IMS enabled conferencing architecture on top of a standard centralized conferencing framework [IP Multimedia Systems (IMS) Infrastructure and Services]," IEEE Communications Magazine, vol. 45, no. 3, pp. 152-159, 2007.

[6] S. E. Deering, "Multicast routing in a datagram internetwork," 1991.

[7] C. Diot, B. N. Levine, B. Lyles, H. Kassem, and D. Balensiefen, "Deployment issues for the IP multicast service and architecture," IEEE Network, vol. 14, no. 1, pp. 78-88, 2000.

[8] C. Luo, W. Wang, J. Tang, J. Sun, and J. Li, "A Multiparty Videoconferencing System Over an Application-Level Multicast Protocol," IEEE Transactions on Multimedia, vol. 9, no. 8, pp. 1621-1632, 2007.

[9] 3gpp, UTRA-UTRAN Long Term Evolution (LTE) and 3GPP System Architecture Evolution (SAE), 2005.

[10] P. Beming, L. Frid, G. Hall, P. Malm, T. Noren, M. Olsson, and G. Rune, "Lte-sae architecture and performance," Ericsson Review, vol. 3, pp. 98-104, 2007.

[11] K. Daoud, P. Herbelin, and N. Crespi, "UFA: Ultra Flat Architecture for high bitrate services in mobile networks," in IEEE 19th International Symposium on Personal, Indoor and Mobile Radio Communications, 2008. PIMRC 2008, 2008, pp. 1-6.

[12] K. Daoud, P. Herbelin, and N. Crespi, "One-Node-Based Mobile Architecture for a Better QoS Control," IFIP Wireless days, 2008.

[13] A. Lung-Yut-Fong, "3G IP Multimedia Subsystem: QoS and Access Convergence," 2006.

[14] Tien A. Le, Quoc T. Tran, and Quang H. Nguyen, Multi-P2P prototype: http://code.google.com/p/multi-p2p/, 2009.

[15] F. Fokus, "Open IMS Core," URL http://www. openimscore. org. 